\title{
Clinical and economic impact of primary hyperoxaluria: a retrospective claims analysis
}

\author{
Lisa Mucha, PhD; Bernd Hoppe, MD; Abigail Silber, MPH; Zheng Wang, BA; Gavin Miyasato, MS; \\ Jeffrey R Skaar, PhD; and Craig Langman, MD
}

\section{What is already known about this subject}

- Primary hyperoxaluria $(\mathrm{PH})$ is a debilitating disease, yet very little is known about the health care utilization and costs associated with specific types of care among $\mathrm{PH}$ patients.

- Characterization of the burden of illness of $\mathrm{PH}$ was challenging before 2018 because there was no ICD-10 code for $\mathrm{PH}$, but with the advent of this code, researchers have been able to discern costs and utilization specific to $\mathrm{PH}$ patients.

\section{What this study adds}

- This study quantifies health care utilization and costs for patients with $\mathrm{PH}$.

- Findings demonstrate that health care costs for $\mathrm{PH}$ patients are often significantly higher than those in a matched cohort without the disease.

\section{Author affiliations}

Lisa Mucha, PhD, and Bernd Hoppe, MD, Dicerna Pharmaceuticals, Lexington MA. Abigail Silber, MPH; Zheng Wang, BA; Gavin Miyasato, MS; and Jeffrey R Skaar, PhD, Trinity Life Sciences, Waltham, MA. Craig Langman, MD, Feinberg School of Medicine, Northwestern University, Chicago, IL.

AUTHOR CORRESPONDENCE:

Lisa Mucha, Imucha@dicerna.com

J Manag Care Spec Pharm. 2022;28(3):316-23

Copyright $\odot 2022$, Academy of Managed Care Pharmacy. All rights reserved.

\section{ABSTRACT \\ BACKGROUND: Primary hyperoxaluria (PH) is a family of rare, life-threatening genetic liver disorders characterized by elevated production and excretion of oxalate. To date, the clinical and economic burden associated with $\mathrm{PH}$ has not been well characterized due to the rarity of the disease and previous chal- lenges with diagnostic coding that prevented proper identification of patients with $\mathrm{PH}$ in claims data.}

OBJECTIVE: To characterize the clinical and economic costs, as well as health care resource utilization (HCRU), associated with $\mathrm{PH}$ relative to a matched cohort of patients without $\mathrm{PH}$.

METHODS: Data from the IQVIA PharMetrics Plus Database were used to conduct a retrospective matched-cohort study to compare differences in clinical characteristics, HCRU, and pharmacy and medical costs in patients with $\mathrm{PH}$ compared with a matched cohort of patients without PH from January 2014 to December 2019.

RESULTS: Overall, 324 patients were included in the $\mathrm{PH}$ cohort and 1,620 patients were in the non-PH cohort. The mean age of $\mathrm{PH}$ patients was 48.1 years, and approximately $58 \%$ of the sample were male. Significantly more patients in the $\mathrm{PH}$ cohort than the non-PH cohort were diagnosed with stage 2 chronic kidney disease (CKD; $3.1 \%$ vs $0.4 \%$, respectively; $P<0.001$ ), stage 3 CKD ( $4.6 \%$ vs $0.5 \% ; P<0.001)$, stage 4 CKD ( $2.5 \%$ vs $0.1 \%$; $P<0.001)$, and stage 5 CKD or end-stage renal disease (ESRD; $2.2 \%$ vs $0.1 \% ; P<0.001$ ). $\mathrm{PH}$ patients had a significantly higher mean Charlson Comorbidity Index composite score than patients in the non- $\mathrm{PH}$ cohort ( 0.79 vs $0.37 ; P<0.001)$. HCRU was significantly higher in patients with $\mathrm{PH}$. The $\mathrm{PH}$ cohort had a significantly higher proportion of patients with at least 1 visit to clinicians specializing in nephrology ( $19 \%$ vs $0.4 \%$, respectively; $P<0.001)$, cardiology ( $22 \%$ vs $12 \% ; P<0.001)$, ophthalmology ( $16 \%$ vs $7 \% ; P<0.001)$, general surgery ( $9 \%$ vs $6 \% ; P=0.011)$, and urology $(65 \%$ vs $6 \% ; P<0.001)$ compared with patients without $\mathrm{PH}$. Mean total annual health care costs in the $\mathrm{PH}$ cohort were $65 \%$ higher than in the non- $\mathrm{PH}$ cohort $(\$ 22,549$ vs $\$ 7,852$, respectively; $P<0.001$ ). Similar results were found for total prescription drug costs ( $\$ 4,125$ vs $\$ 2,464 ; P=0.012)$.

CONCLUSIONS: Despite the rarity of $\mathrm{PH}$, patients with this disease incur substantial clinical and economic burden and may cause financial strain on the health care system. Additional research is warranted to understand the economic and clinical burden of $\mathrm{PH}$ stratified by the 3 subtypes of the disease. 
Primary hyperoxaluria (PH) is a family of rare, life-threatening genetic liver disorders characterized by elevated production and excretion of oxalate., ${ }^{1,2}$ Three subtypes of $\mathrm{PH}$ have been identified, each caused by a genetic mutation and resulting in a distinct enzyme deficiency; all $\mathrm{PH}$ subtypes ultimately lead to the overproduction of oxalate in the liver, as well as an increase in the excretion of oxalate by the kidneys. ${ }^{1,2}$ Excess oxalate combines with calcium in the kidneys to form calcium oxalate, the primary component of kidney stones; consequently, patients with $\mathrm{PH}$ often experience recurrent kidney stones starting at a young age, with the mean age of PH symptom onset around 7.5 years (median: 4.1 years) $)^{3,4}$

An additional outcome of excessive oxalate can be the development of calculi throughout the urinary tract (urolithiasis) and kidneys (nephrolithiasis), obstruction of the renal tubules, ${ }^{5}$ and a progressive increase of calciumoxalate deposition in the kidneys (nephrocalcinosis). ${ }^{6}$ A chronic inflammatory process induced by the internalization of calcium oxalate crystals in the kidneys may lead to chronic kidney disease (CKD) or end-stage renal disease (ESRD) in tandem with systemic oxalosis, a process by which calcium oxalate crystals are deposited throughout various organ systems..$^{2,5}$ Systemic oxalosis often affects bone, skin, retina, myocardium, blood vessels, and even the central nervous system, potentially leading to serious complications or death if left untreated. ${ }^{2}$

The prevalence of $\mathrm{PH}$ is estimated to be less than 3 diagnosed cases per 1,000,000 individuals. ${ }^{1}$ In contrast, whole-genome sequencing studies have suggested that the prevalence of $\mathrm{PH}$ (based on known mutations) is approximately 1 case in 58,000 individuals, suggesting that many cases are undiagnosed or misdiagnosed; thus, the prevalence of $\mathrm{PH}$ may be greatly underestimated due to some patients presenting with relatively mild symptoms or becoming symptomatic only later in life. ${ }^{1}$

The management of $\mathrm{PH}$ varies depending on the type, severity, and progression of the disease, ${ }^{2}$ with only 1 pharmacologic treatment (lumasiran) approved by the US Food and Drug Administration for the treatment of $\mathrm{PH}$ type $1 .^{7}$ Approaches to treatment for patients in early stages of PH also may consist of increasing fluid intake and pyridoxine (vitamin B6 in PH1) to help dilute and reduce endogenous oxalate production (B6) and thus urinary oxalate. ${ }^{5,8}$ Administration of potassium or sodium citrate may also be beneficial for urine alkalinization and an increase of urinary citrate excretion, thereby inhibiting calcium oxalate crystallization. ${ }^{8} \mathrm{PH}$ patients may also progress to ESRD requiring dialysis, making isolated kidney transplantation or combined liver-kidney transplantation, in selected cases, necessary. ${ }^{2,6}$
Because of the rarity of $\mathrm{PH}$ and, until recently, lack of diagnostic coding to enable identification of this specific diagnosis through claims data analyses, the clinical and economic burden of PH has not been well documented. However, patients with PH can now be identified by International Classification of Diseases, Tenth Revision, Clinical Modification (ICD-10-CM) codes so assessing the burden directly associated with $\mathrm{PH}$ is now possible. Thus, the objective of the present study was to characterize the clinical and economic costs and health care resource utilization (HCRU) associated with diagnosed $\mathrm{PH}$ relative to a matched cohort of patients without the disease or any official diagnosis of $\mathrm{PH}$.

\section{Methods}

\section{STUDY DESIGN AND DATA SOURCE}

A retrospective, matched cohort study using commercial claims data from the IQVIA PharMetrics Plus Database was performed. The PharMetrics Plus Database contains demographic information, health plan enrollment, medical and pharmacy claims, characteristics of HCRU (including place of service), and ICD-10-CM codes for over 140 million Americans with health insurance. Differences in clinical characteristics, HCRU, and pharmacy and medical costs were evaluated in patients with $\mathrm{PH}$ compared with a matched cohort of patients without PH from January 2014 to December 2019.

\section{STUDY POPULATION SELECTION}

Two cohorts were constructed for this analysis. The first was the PH cohort, which included patients of any age who had at least 1 claim reported on or after October 2018 with a PH diagnosis (ICD-10-CM code E72.53) and were continuously enrolled in both medical and prescription benefits for at least 1 year before and following the diagnostic code. Before October 2018, the hyperoxaluria code included primary and secondary types, so the ICD code was only used after October 2018, when it became specific to $\mathrm{PH}$.

The second cohort consisted of non-PH patients. For this cohort, a $5 \%$ random sample of patients of any age who did not have any of the diagnoses (including PH or calculi [stones]) was drawn from the dataset (Supplementary Table 1, available in online article). However, in order to mitigate bias by the exclusion of calculi in the non-PH cohort, an additional 5\% random sample of patients with calculi but no PH diagnosis was drawn and then restored to the non-PH cohort. Patients who were not continuously enrolled in medical and prescription benefits for the study period were excluded. The remaining patients were then 


\section{FIGURE 1 Sample Construction Flow Chart}

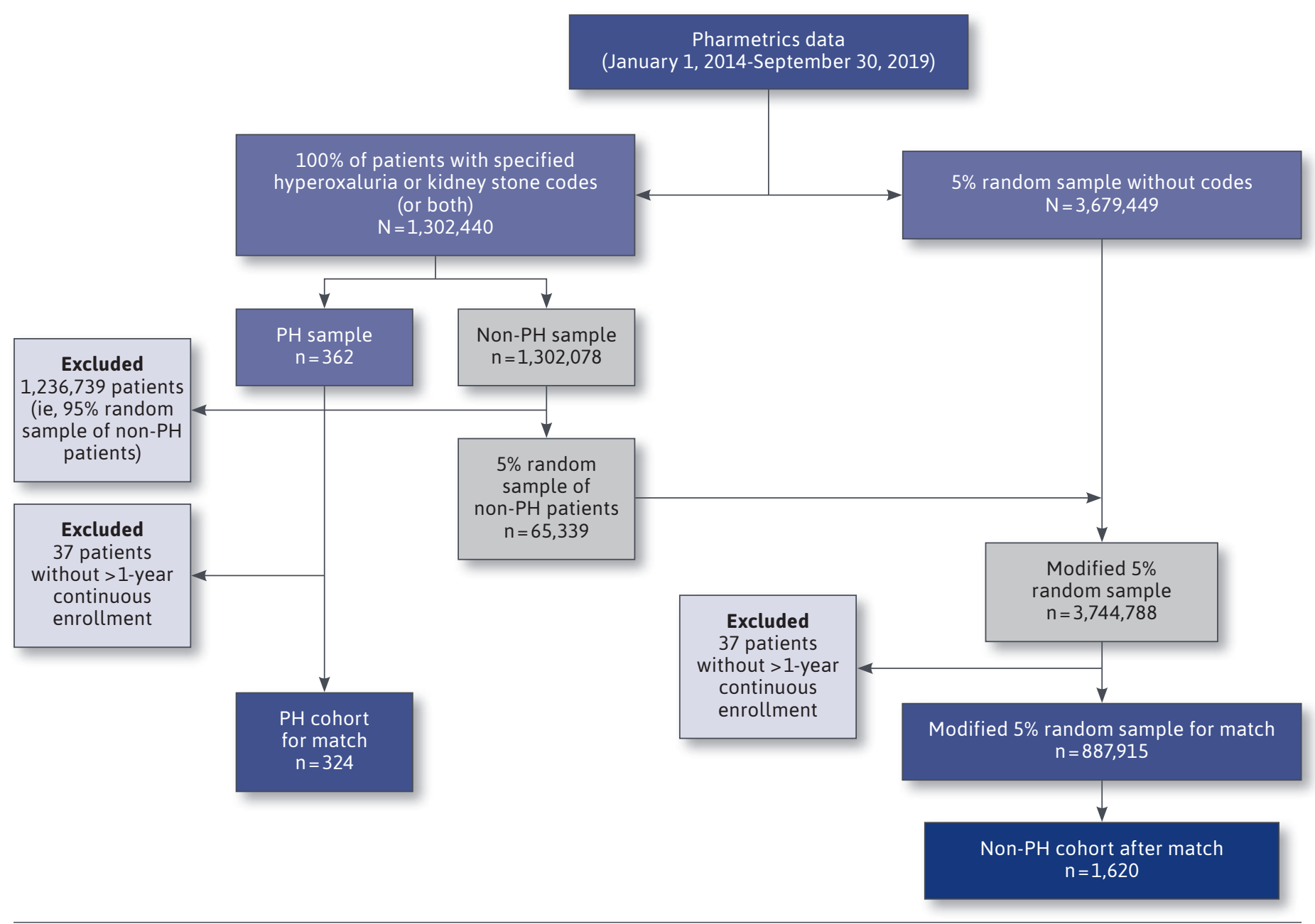

$\mathrm{PH}=$ primary hyperoxaluria .

randomly matched to patients with $\mathrm{PH}$ (5 patients without $\mathrm{PH}$ to 1 patient with $\mathrm{PH}$ ) based on age, sex, and insurance type. ${ }^{9}$ Patients in the non-PH cohort who did not match to a patient with $\mathrm{PH}$ were excluded from the analyses.

\section{PATIENT DEMOGRAPHICS AND CLINICAL OUTCOMES}

Patient demographics such as age, sex, race, region, and insurance type were recorded and compared between patients in the PH and non-PH cohorts. Clinical outcomes of interest included occurrence of kidney stones and urinary tract infections, CKD stages, acute kidney failure, kidney or liver transplantation, and dialysis.

Comorbidities of patients in the $\mathrm{PH}$ and non-PH cohorts were compared using the Quan et al adapted version of the
Charlson Comorbidity Index (CCI). ${ }^{10}$ The index consists of 12 diseases weighted to sum to a score ranging from 0-24, with a higher score indicating a greater disease burden. For the present analyses, the percentage of patients with a diagnosis for each comorbidity was calculated in addition to the mean CCI scores for patients in the PH and non-PH cohorts.

\section{HEALTH CARE RESOURCE UTILIZATION}

HCRU was defined as the total number of inpatient, outpatient, emergency department, and physician office visits, as well as prescription drug claims for a 12-month period after the index date. The annual number of patient visits to select physician specialties, including nephrology, cardiology, ophthalmology, pediatrics, general surgery, and urology, 


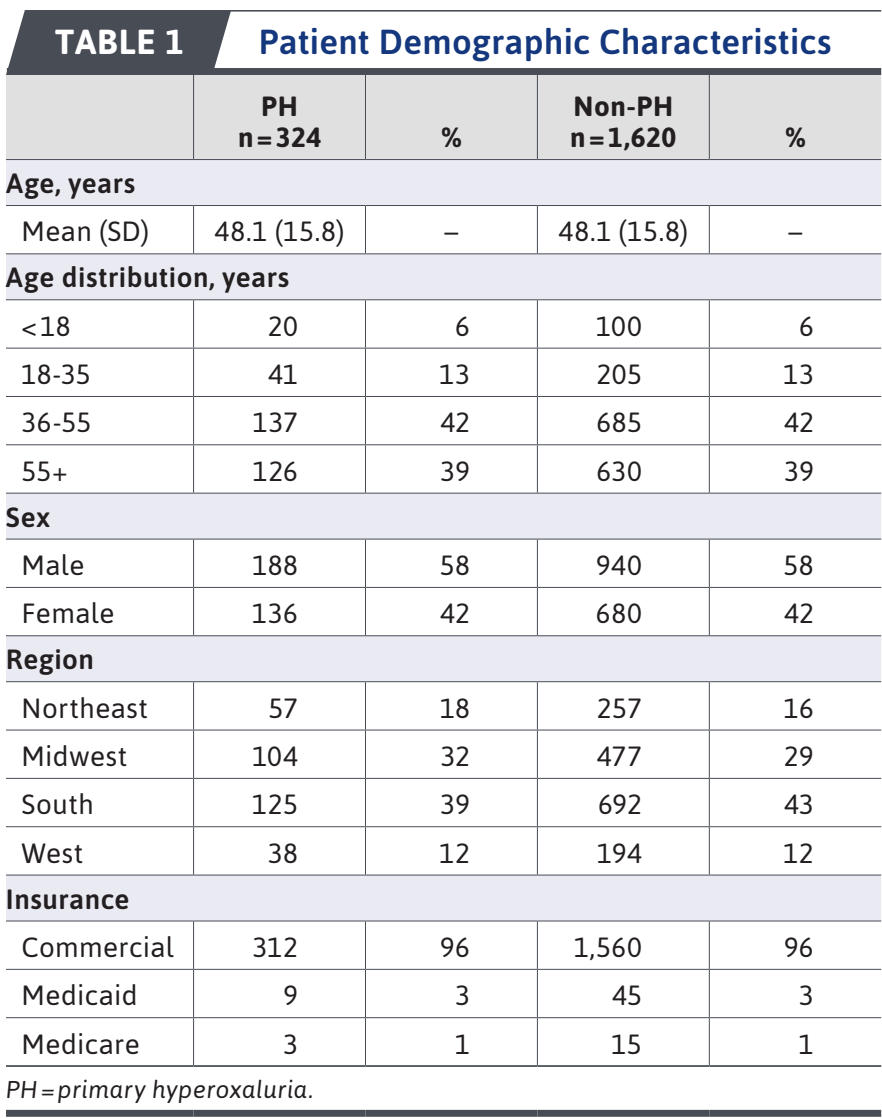

were also reported based on the number of patients with at least 1 encounter in any of those specialty areas.

\section{HEALTH CARE COSTS}

Total medical and prescription drug costs were adjusted to the 2019 medical care component of the Consumer Price Index and reflected the 12-month costs across all patients in each cohort.

Mean prescription costs were compared for patients in the $\mathrm{PH}$ and non-PH cohorts by examining the 15 costliest prescription drug classes used by patients in the $\mathrm{PH}$ cohort. In addition to these 15 medication classes, the mean and median costs of opioid and narcotic analgesics (as a drug class) were examined. Although opioid use has yet to be investigated in patients with $\mathrm{PH}$, use of opioid or narcotic analgesic drugs (as a class) was of interest, since individuals with PH reportedly deal with high levels of pain. ${ }^{11}$

\section{STATISTICAL ANALYSIS}

All analyses were performed using SAS/STAT 14.3 (SAS Institute Inc.) and $\mathrm{R}$ 3.6.2 (R Foundation). Categorical variables were reported as percentages, while continuous variables were reported as means and SDs. The median value is only shown for total health care costs. Significant differences between cohorts were examined using the bootstrap t-test and Wilcoxon rank sum test. For categorical variables, the chi square test was used to test for statistically significant differences in proportions.

\section{Results}

\section{PATIENT DEMOGRAPHICS AND CLINICAL OUTCOMES}

A total of 1,944 patient records (hereafter referred to as "patients") were included in the study ( $\mathrm{PH}$ cohort, $\mathrm{n}=324$; non-PH cohort, $\mathrm{n}=1,620$; Figure 1). Most $\mathrm{PH}$ patients (96\%) had commercial insurance, and $4 \%$ had Medicaid or Medicare (Table 1). The mean (SD) age of patients was 48.1 (15.8) years, and approximately $58 \%$ of the sample was male.

Significantly more patients in the PH cohort than the non-PH cohort were diagnosed with stage 2 CKD (3.1\% vs $0.4 \%$, respectively; $\mathrm{P}<0.001)$, stage $3 \mathrm{CKD}(4.6 \%$ vs $0.5 \%$; $\mathrm{P}<0.001)$, stage 4 CKD $(2.5 \%$ vs $0.1 \% ; \mathrm{P}<0.001)$, and stage 5 CKD or ESRD (2.2\% vs $0.1 \%$; $\mathrm{P}<0.001$; Table 2$)$. A greater proportion of patients in the $\mathrm{PH}$ cohort than the non-PH cohort had a history of kidney stones before study entry ( $35 \%$ vs $0.9 \%$, respectively; $P<0.001$ ). Among those patients who had a stone in the 12-month study period, a greater proportion of patients in the $\mathrm{PH}$ cohort than in the non- $\mathrm{PH}$ cohort had 2 or more stones (59\% vs $24 \%$, respectively; $\mathrm{P}<0.001)$. Despite the sample being more heavily representative of patients in lower CKD stages, a small proportion of $\mathrm{PH}$ patients underwent organ transplantation during the study (2.2\% kidney, 1.2\% liver); however, no patients in the non-PH cohort underwent kidney or liver transplantation during the study period.

A significantly greater proportion of patients in the $\mathrm{PH}$ cohort relative to the non-PH cohort were diagnosed with 1 or more of the following comorbidities via presence of the disease-specific ICD-10-CM code on a claim (all $\mathrm{P}<0.001$ ): peripheral vascular disease $(5.2 \%$ vs $2.0 \%)$, cerebrovascular disease $(4.3 \%$ vs $1.4 \%)$, mild liver disease (9.9\% vs $3.5 \%$ ), diabetes without chronic complications (18\% vs $10 \%)$, hemiplegia or paraplegia ( $2.2 \%$ vs $0.2 \%$ ), and moderate or severe renal disease (10\% vs $1.3 \%$; Supplementary Table 2 , available in online article). Patients in the PH cohort had a significantly higher mean CCI composite score, indicating greater disease burden, than patients in the non-PH cohort (0.79 vs 0.37 , respectively; $\mathrm{P}<0.001$ ). 


\begin{tabular}{|c|c|c|c|}
\hline & $\begin{array}{l}\mathrm{PH}, \% \\
\mathrm{n}=324\end{array}$ & $\begin{array}{c}\text { Non-PH, \% } \\
n=1,620\end{array}$ & $P$ value \\
\hline History of kidney stones & 35 & 0.9 & $<0.001$ \\
\hline$\geq 1$ kidney stone occurrence in the study period & 80 & 3 & $<0.001$ \\
\hline 22 occurrences ${ }^{a}$ & 59 & 24 & $<0.001$ \\
\hline \multicolumn{4}{|l|}{ CKD, by stage } \\
\hline Stage 1 & 0.6 & 0.2 & 0.273 \\
\hline Stage 2 & 3.1 & 0.4 & $<0.001$ \\
\hline Stage 3 & 4.6 & 0.5 & $<0.001$ \\
\hline Stage 4 & 2.5 & 0.1 & $<0.001$ \\
\hline Stage 5 or ESRD & 2.2 & 0.1 & $<0.001$ \\
\hline Acute kidney failure & 6.5 & 1.1 & $<0.001$ \\
\hline Kidney transplantation & 2.2 & 0.0 & $<0.001$ \\
\hline iver transplantation & 1.2 & 0.0 & $<0.001$ \\
\hline$\geq 1$ UTI & 18 & 3.1 & $<0.001$ \\
\hline$\geq 2$ UTIs among patients with $\geq 1$ UTI & 29 & 10 & 0.013 \\
\hline
\end{tabular}

\section{HEALTH CARE RESOURCE UTILIZATION}

HCRU was significantly higher in the $\mathrm{PH}$ cohort across most types of care in the aggregate included in this study. When prespecified specialty care was examined individually, the PH cohort had a significantly higher proportion of patients with at least 1 visit to clinicians specializing in nephrology $(19 \%$ vs $0.4 \%$, respectively; $\mathrm{P}<0.001)$, cardiology (22\% vs $12 \%$; $P<0.001)$, ophthalmology (16\% vs $7 \%$; $P<0.001$ ), general surgery (9\% vs $6 \% ; \mathrm{P}=0.011$ ), and urology (65\% vs $6 \%$; $\mathrm{P}<0.001$; Table 3). The lone exception was visits to a pediatrician; no significant between-cohort difference was observed $(P=0.139)$, which may have been attributable to an insufficient pediatric sample size in this analysis.

\section{HEALTH CARE COSTS}

Regardless of setting, all mean annual health care costs were significantly higher for patients in the $\mathrm{PH}$ cohort than in the non-PH cohort (Table 3). For total health care costs, mean and median costs were significantly higher in the $\mathrm{PH}$ cohort; mean costs were 2.9 times higher in the $\mathrm{PH}$ cohort than in the non-PH cohort $(\$ 22,529$ vs $\$ 7,852$, respectively; $\mathrm{P}<0.001$ ), and median costs were 6.5 times higher ( $\$ 11,017$ vs $\$ 1,685 ; \mathrm{P}<0.001)$. The differentials for individual types of care continued to exhibit a pattern of higher utilization in the PH cohort. The highest mean costs in the 12-month study period for the $\mathrm{PH}$ cohort compared with the non-PH cohort were in outpatient visits (\$13,894 vs $\$ 3,784$, respectively; $\mathrm{P}<0.001)$ followed by inpatient visits $(\$ 4,530$ vs $\$ 1,605 ; \mathrm{P}<0.001)$. The percentage difference in costs for care was consistently higher in the $\mathrm{PH}$ cohort and ranged from $40 \%$ higher for prescription drugs to $77 \%$ higher for outpatient surgery.
Prescription drug annual costs were reported in the aggregate and for selected individual classes. The mean total cost of prescription drugs was 1.7 times higher for patients in the $\mathrm{PH}$ cohort than for patients in the non-PH cohort ( $\$ 4,125$ vs $\$ 2,464$, respectively; $\mathrm{P}<0.001)$. Among the top 15 most expensive prescription drug categories used by patients in the $\mathrm{PH}$ cohort, several significant betweencohort differences were observed (Figure 2).

The mean costs of prescriptions were significantly higher for patients in the $\mathrm{PH}$ cohort compared with the non-PH cohort for antiarthritics $(\$ 1,077$ vs $\$ 278$, respectively; $\mathrm{P}=0.020)$, anticoagulants ( $\$ 107$ vs $\$ 35 ; P=0.012$ ), lipotropics ( $\$ 80$ vs $\$ 38$; $\mathrm{P}<0.001)$, adrenergics (\$69 vs $\$ 4$; $\mathrm{P}=0.012$ ), ophthalmic preparations (\$69 vs $\$ 18 ; P=0.004)$, and electrolytes/miscellaneous nutrients (\$56 vs $\$ 2 ; \mathrm{P}<0.001)$.

While not included in the top 15 most expensive drug classes, a significantly greater proportion of patients in the $\mathrm{PH}$ cohort were prescribed opioids or narcotic analgesics compared with patients in the non-PH cohort (47\% vs $15 \%$, respectively; $\mathrm{P}<0.001)$.

\section{Discussion}

To our knowledge, this is the first study to describe HCRU and the overall economic burden of patients with $\mathrm{PH}$ compared with those with no diagnostic claim for PH. Overall, our results indicate that patients with $\mathrm{PH}$ generally used more health care resources and generated greater health care and prescription drug annual costs relative to their matched non-PH counterparts. As expected, a greater proportion of patients with $\mathrm{PH}$ experienced 1 or more kidney stone events and had a diagnosis of stages 2-5 CKD. As expected, a greater number of patients in the $\mathrm{PH}$ cohort 


\section{TABLE 3 Health Care Resource Utilization and Costs}

\begin{tabular}{|c|c|c|c|}
\hline & $\begin{array}{c}\text { PH } \\
n=324 \\
\text { Mean (SD) }\end{array}$ & $\begin{array}{c}\text { Non-PH } \\
n=1,620 \\
\text { Mean (SD) }\end{array}$ & $P$ value \\
\hline \multicolumn{4}{|l|}{ Health care resource utilization } \\
\hline Outpatient visits & $23(21)$ & 9 (13) & $<0.001$ \\
\hline Inpatient visits & $0.8(4.2)$ & $0.2(2.2)$ & $<0.001$ \\
\hline Emergency department visits & $0.7(1.4)$ & $0.2(0.6)$ & $<0.001$ \\
\hline Physician office visits & $13(14)$ & $6 \quad(8)$ & $<0.001$ \\
\hline \multicolumn{4}{|c|}{ Patients with $\geq 1$ visit to any specialist, $\%$} \\
\hline Urologist & 65 & 6 & $<0.001$ \\
\hline Cardiologist & 22 & 12 & $<0.001$ \\
\hline Nephrologist & 19 & 0.4 & $<0.001$ \\
\hline Ophthalmologist & 16 & 7 & $<0.001$ \\
\hline General surgeon & 9 & 6 & 0.011 \\
\hline Pediatrician & 7 & 5 & 0.139 \\
\hline \multicolumn{4}{|l|}{ Health care costs, $\$$} \\
\hline Total mean costs (SD) & $22,549(36,716)$ & $7,852(23,552)$ & $<0.001$ \\
\hline Total median costs & 11,017 & 1,685 & $<0.001$ \\
\hline \multicolumn{4}{|l|}{ Mean costs by type of care } \\
\hline Outpatient visits & $13,894(19,444)$ & $3,784 \quad(8,636)$ & $<0.001$ \\
\hline Inpatient visits & $4,530(18,176)$ & $1,605(12,282)$ & $<0.001$ \\
\hline Prescription drugs & $4,125(10,620)$ & $2,464(13,311)$ & $<0.001$ \\
\hline Outpatient surgery & $3,379 \quad(6,514)$ & $785 \quad(3,180)$ & $<0.001$ \\
\hline Patient cost-share amount & $2,737 \quad(2,728)$ & $1,145 \quad(1,927)$ & $<0.001$ \\
\hline Physician office visits & $1,759 \quad(2,126)$ & $684 \quad(1,014)$ & $<0.001$ \\
\hline Emergency department visits & $651(1,250)$ & (769) & $<0.001$ \\
\hline
\end{tabular}

had renal or liver disease than in the non-PH cohort. What was unexpected, however, was that the comorbidity most frequently identified as part of the components of the CCI scores for patients with PH was diabetes without chronic complications (CCI does not stratify by type I or II; $18 \%$ of patients with $\mathrm{PH}$ vs $10 \%$ of non $\mathrm{PH}$ patients; $\mathrm{P}<0.001)$. Further research is required to determine if there is a relationship, causal or otherwise, between diabetes and $\mathrm{PH}$.

Our findings also suggest that a greater proportion of patients with $\mathrm{PH}$ consulted specialists in nephrology, cardiology, ophthalmology, and urology compared with those without $\mathrm{PH}$. While this may be a function of needed treatment for comorbidities and the sequelae of $\mathrm{PH}$ itself, these results were consistent with other outcomes in this study, indicating that patients with PH use more health care resources and generate greater overall costs than their matched non-PH controls. While higher rates of utilization among patients in the $\mathrm{PH}$ cohort were expected for specialty areas related to PH (eg, urology), the elevated utilization in other areas, such as cardiology and ophthalmology, were unexpected.
Additional research will be needed to explore this further.

Results of this study demonstrated the substantial economic costs associated with $\mathrm{PH}$. Total health care annual costs were substantially higher in the $\mathrm{PH}$ cohort, indicating that the burden of $\mathrm{PH}$ is pervasive across different health care settings. Total prescription drug costs were also higher for $\mathrm{PH}$ patients compared with non-PH patients, despite many of the classes, including generic agents, and no approved therapeutics for $\mathrm{PH}$ being available during the data collection period. The impact of available (such as lumasiran) or upcoming pharmacologic treatments for $\mathrm{PH}$ on prescription drug costs should be the subject of additional research. Additionally, a greater proportion of patients in the $\mathrm{PH}$ cohort had claims for opioid and narcotic analgesics than in the non-PH cohort. While this may be related to pain management of renal sequelae, such as kidney stones, additional research is needed to establish a causal link.

\section{LIMITATIONS}

As with all claims database analyses, there are inherent limitations to the present study. ICD codes before October 2018 did not distinguish between primary and secondary hyperoxaluria. For this reason, our analyses were limited to patients with the PH specific ICD code appearing after October 2018. It is also assumed that the coding was accurate, although the possibility of inaccurate and inconsistent coding exists. Furthermore, the ICD-10 code did not allow stratification by $\mathrm{PH}$ subtypes 1 , 2 , and 3.

Our sample contained a very low proportion of pediatric patients (aged $<18$ years) and renally compromised patients; therefore, the true clinical and economic burden of PH may have 


\section{FIGURE 2 Prescription Drug Costs ${ }^{\mathrm{a}}$ by Class}

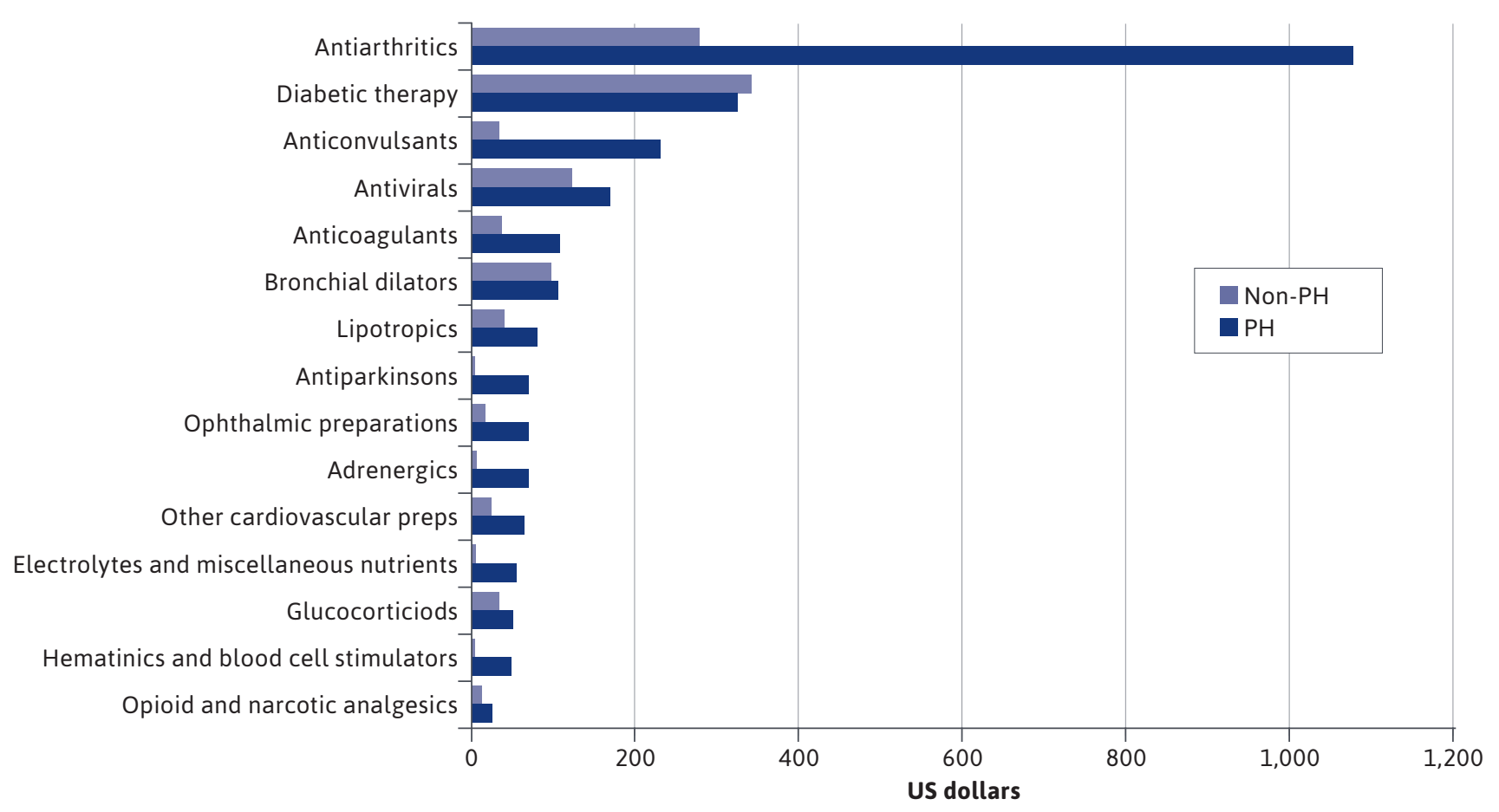

aSignificant differences are: Antiarthritics $P=0.02$, Anticonvulsants $P=0.072$, Anticoagulants $P=0.012$, Lipotropics $P<0.001$, Ophthalmic preparations $P=0.004$, Adrenergics $P=0.012$, Other cardiovascular preps $P=0.036$, Electrolytes/misc. nutrients $P<0.001$, Hematinics $P=0.056$, and Opioid/narcotic analgesic $P=0.032$. $\mathrm{PH}=$ primary hyperoxaluria.

been underestimated. It is not clear why claims for this rare condition were not seen in a greater number of pediatric patients in this claims database. Also, while the likelihood of including undiagnosed $\mathrm{PH}$ patients in the non-PH group was low, it may have happened. Given the lack of a validated algorithm to accurately identify undiagnosed patients, it was not possible to detect them via claims and exclude them from the non-PH group. If this did occur, it may have been so few patients that fell into the initial 3.7 million claims reviewed for the non-PH group that their influence on the results would be negligible at best.

Results of the current study were based primarily on commercial claims data, so our findings may not be generalizable to patients with noncommercial payer types (eg, Medicare and Medicaid). The small proportion of Medicare patients also would have been an indicator of underrepresentation of patients with ESRD. Inclusion in this study required continuous enrollment, which may have excluded patients who switched insurance providers.
Finally, this study did not link prescription drugs to the patients' ICD-10-CM codes, so the indication that the prescriptions were written for was not specified.

\section{Conclusions}

The results presented here suggest that PH leads to a substantial clinical and economic burden for patients and health care systems. Although PH is a rare disease, patients were found to incur substantial costs across most types of health care. This demonstrated that a small number of diagnosed $\mathrm{PH}$ patients can still incur significant costs and clinical outcomes, such as chronic comorbidities and sequelae commonly found in PH (eg, kidney stones). Further research is needed to evaluate the burden of PH stratified by subtype and to assess the impact of advanced disease stage on clinical and economic outcomes. 


\section{DISCLOSURES}

Funding for this research was provided by Dicerna Pharmaceuticals. Mucha and Hoppe are employed by Dicerna Pharmaceuticals. Silber Miyasoto, Skaar, and Wang are employed by Trinity Life Sciences, which was contracted by Dicerna Pharmaceuticals to conduct the study analysis. Langman is consultant to Dicerna Pharmaceuticals.

This study was presented as a poster at the AMCP Nexus 2020 (virtual), October 19-23, 2020, and American Society of Nephrology 2020 (virtual), October 19-25, 2020.

\section{REFERENCES}

1. Hopp K, Cogal AG, Bergstralh EJ, et al. Phenotype-genotype correlations and estimated carrier frequencies of primary hyperoxaluria. J Am Soc Nephrol. 2015;26(10):2559-70.
2. Cochat P, Rumsby G. Primary hyperoxaluria. N Engl J Med. 2013;369(7):649-68.

3. Bhasin B, Ürekli HM, Atta MG. Primary and secondary hyperoxaluria: understanding the enigma. World J Nephrol. 2015;4(2):235-44.

4. Sas DJ, Enders FT, Mehta RA, et al. Clinical features of genetically-confirmed patients with primary hyperoxaluria identified by clinical indivation versus familial screening. Kidney Int. 2021;97(4):786-92.

5. Ben-Shalom E, Frishberg Y. Primary hyperoxalurias: diagnosis and treatment. Pediatr Nephrol. 2015;30(10):1781-91.

6. Hoppe B. An update on primary hyperoxaluria. Nat Rev Nephrol. 2012;8(8):467-75.

7. Oxlumo. Prescription information. Alnylam Pharmaceuticals, Inc; 2020. Accessed January 8, 2022. https://www. accessdata.fda.gov/drugsatfda_docs/ label/2020/214103lbl.pdf
8. Harambat J, Fargue S, Bacchetta J, Acquaviva C, Cochat P. Primary hyperoxaluria. Int J Nephrol. 2011;2011:864580.

9. Shoffstall AJ, Gaebler JA, Kreher NC, et al. The high direct medical costs of Prader-Willi syndrome. J Pediatr. 2016;175:137-43.

10. Quan H, Li B, Couris CM, et al. Updating and validating the Charlson Comorbidity Index and score for risk adjustment in hospital discharge abstracts using data from 6 countries. Am J Epidemiol. 2011;173(6):676-82.

11. Cochat P, Groothoff J. Primary hyperoxaluria type 1: practical and ethical issues. Pediatr Nephrol. 2013;28(12):2273-81. 